\title{
Ethik im Gesundheitswesen - Fall einer psychisch Kranken mit einer unheilbar körperlichen Krankheit mit Sterbewunsch
}

G. Ebner

Korrespondenz:

Dr med. Gerhard Ebner

Psychiatriezentrum Schaffhausen

Breitenaustrasse 124

CH-8200 Schaffhausen
Eine ältere Patientin, die seit Jahren ambulant und stationär bei uns behandelt wurde, wird zunehmend wegen eines Karzinoms pflegebedürftig und auf eine Pflegeabteilung verlegt. Sie wird intensiv psychologisch von einem klinischen Psychologen betreut. Die Pflege ist professionell ausgelegt, indem sie einerseits die funktionell pflegerischen Aspekte, andererseits aber auch die besondere psychiatrische Situation anerkennt, die Betreuung durch den Psychologen unterstützt und einen regelmässigen Austausch zwischen den betreuenden Berufsgruppen (Arzt, Psychologe, Pflege) erwartet.

Im Laufe der Gespräche mit dem Psychologen äussert die Patientin grosse Ängste auf ihre zunehmende Pflegebedürftigkeit hin, ihre Abhängigkeit von anderen, aber auch vor den Schmerzen, die jetzt schon sehr ausgeprägt sind, und Angst vor dem nahenden Tod, in erster Linie aber vor der bis dahin in Aussicht stehenden quälenden Zeit. In diesem Zusammenhang bittet sie den Psychologen, ihr über eine Sterbehilfeorganisation zu ermöglichen, dass sie mit Hilfe eines assistierten Suizides aus dem Leben scheiden kann, um sich all die drohenden Belastungen so zu ersparen. Der Psychologe kommt mit diesem Anliegen zum Chefarzt.

\section{Krankheitsgeschichte}

Die Patientin leidet seit vielen Jahren unter rezidivierenden depressiven Episoden; zwischen diesen Episoden kommt es zwar zu deutlicher Besserung, jedoch nicht zu einer restitutio ad integrum; die Patientin ist im Rahmen der Residualsymptomatik in ihrer Lebensführung eingeschränkt, kann aber mit ihrem Partner zusammen den Haushalt führen.

Während der körperlichen Erkrankung ist die depressive Symptomatik eher im Hintergrund, die Patientin ist in der Lage, differenziert ihre Situation zu reflektieren; sie wird von uns als urteilsfähig in bezug auf die Frage des assistierten Suizides eingeschätzt; sie befindet sich nicht in einer schweren Krankheitsphase. So macht sie den Suizidwunsch nun auch nicht an der bei Depressiven typischen Selbstwertproblematik, allgemeinen Sinnlosigkeitsgefühlen und allgemeiner Hoffnungslosigkeit, die mit der objektiv gesehenen Lebenssituation nicht in Einklang zu bringen sind, fest, sondern sehr konkret an der infausten Prognose und den drohenden vom Arzt auch anerkannten Belastungen. Sie ist in der Lage, die Bedeutung eines solchen Schrittes für ihren Partner und die Bedeutung bei zunehmender Pflegebedürftigkeit für sie beide zu erkennen.

\section{Beziehungssituation}

Neben ihrem Partner stehen in erster Linie die professionellen Beziehungen durch Mitarbeiter des Psychiatriezentrums im Vordergrund; das Psychiatriezentrum stellt so für sie einen $\mathrm{Zu}$ fluchtsort dar, zu dem sie Vertrauen hat, und sie wünscht, von uns bis zum Tode betreut zu werden. Eine Verlegung nach Hause erscheint aus Sicht der Patientin, ihres Partners wie des Psychiatriezentrums nicht sinnvoll. Die Pflege unterstützt das Anliegen, die Patientin bis zum Tode zu begleiten. Sie sieht es als ihre Aufgabe an, dieser Frau beizustehen und zeigt grosses Engagement in der Arbeit. So werden zwei feste Bezugspersonen definiert, die in Zusammenarbeit mit dem Psychologen und dem Arzt die Betreuung übernehmen. Der Psychologe ist ebenfalls engagiert, indem er die Patientin täglich sieht und in seiner Ferienabwesenheit regelmässig mit ihr telefoniert mittels eines Handys, das ihr zu diesem Zweck zur Verfügung gestellt wurde.

\section{Psychodynamische Überlegungen und Stellungnahme des Psychologen}

Sowohl von der Lebensgeschichte wie auch in ihrer Krankheit erlebt sich die Patientin als abhängig von anderen und wenig fähig, eigene Bedürfnisse als gerechtfertigt zu erkennen und zu 
verwirklichen. In der Klinik wurde sie immer wieder zum schwerkranken Objekt ärztlich pflegerischer Behandlung mit allen Einschränkungen der Autonomie. Das Ziel der Arbeit des Psychologen besteht darin, mit der Patientin ihre eigenen Bedürfnisse zu formulieren und zu erarbeiten, wo sie diese allenfalls auch jetzt in begrenztem Umfang verwirklichen könnte. Er sieht den Wunsch der Patientin, mit Hilfe einer Sterbehilfeorganisation ihr Leiden abzukürzen, bei aller Problematik auch als Ausdruck dafür an, dass die Patientin sich von Abhängigkeitsbeziehungen lösen und ihr Leben bei einer existentiellen Frage aktiv in die Hand nehmen möchte. Eine kategorische Ablehnung ihres Wunsches - auch wenn aus innerbetrieblichen wie ausserbetrieblichen politischen Überlegungen und ethischen Gründen nachvollziehbar - würde er als Verrat an der Patientin ansehen.

\section{Stellungnahme des behandelnden Arztes}

Der Arzt anerkennt die Argumentation des Psychologen und die Legitimität des Wunsches der Patientin und bestätigt deren Urteilsfähigkeit in bezug auf diese Frage. Der Sterbehilfediskussion gegenüber zeigt er sich offen, undogmatisch. Er betont aber die besondere Verantwortung der Psychiatrie, vor allem, da es sich um einen Präzedenzfall mit allen Konsequenzen handeln könnte.

\section{Stellungnahme der Pflege}

Auch die Pflege anerkennt die Legitimität des Wunsches, sie zeigt sich offen und undogmatisch bei der Diskussion über die Sterbehilfe, sieht ihre Aufgabe aber nicht darin, der Patientin diesen Wunsch nun in der Klinik in jedem Fall zu gewähren. Sie sieht ihre Primäraufgabe darin, alles mögliche zu tun, um der Patientin unnötige Belastungen $\mathrm{zu}$ ersparen, damit die Frage des assistierten Suizids in den Hintergrund treten würde. Ansonsten verweist sie auf die Verantwortung der Ärzte in der Entscheidung.

\section{Stellungnahme des Partners}

Dieser bekundet die Unterstützung des Entscheides der Ärzte, wie auch immer er ausfallen würde; für ihn ist klar, dass er in jedem Fall seine Partnerin unterstützen und sich an die ärztlichen Empfehlungen halten werde.

\section{Stellungnahme des Chefarztes}

Der Chefarzt anerkennt ebenfalls die Legitimität des Wunsches der Patientin und die Urteilsfähigkeit in bezug auf diese Frage. Er hatte bisher eine offene Haltung in bezug auf den assistierten Suizid eingenommen und war in Opposition zu der herrschenden Meinung unter seinen Psychiaterkollegen getreten, indem er sich zwar nicht «für» den assistierten Suizid bei psychisch Kranken aussprach, jedoch für die gleichen Rechte bei psychisch Kranken im Falle einer körperlichen Erkrankung mit infauster Prognose. Die Tatsache allein, dass jemand psychisch krank sei und in bezug auf die Frage des assistierten Suizides voll urteilsfähig, dürfe nicht als alleiniges Argument dienen, ihn dieser Gruppe zu verwehren. Gleichzeitig ist er sich der Rolle bewusst, in die er wie auch das Psychiatriezentrum kommen würden: Präzedenzfall mit Gefahr der Schädigung des Rufes des Psychiatriezentrums; vor allem aber Aufforderungscharakter für andere psychisch Kranke, einer hochgefährdeten Gruppe für Suizide. Er sieht das kollektive Interesse der psychiatrischen Institution durch eine Gewährung gefährdet. Insbesondere anerkennt er weitgehend die Argumente der Kritiker des Entscheides des Zürcher Stadtrates, die betonen, dass sich dieser Entscheid fatal auf Kranke auswirken könnte: Druck auf Pflegebedürftige, sich zu suizidieren, um belastenden Pflegesituationen auszuweichen. Ferner drastische Erhöhung des Suizidrisikos im ganzen Psychiatriezentrum.

\section{Lösungsmöglichkeiten}

1. Dem Wunsch der Patientin wird stattgegeben. Sie erfüllt die Voraussetzungen für die Möglichkeit eines assistierten Suizids (Leiden an einer somatischen Erkrankung mit infauster Prognose, zu erwarten sind grosse Belastung bis zum Tod, Urteilsfähigkeit in bezug auf diese Frage). Hierbei gäbe es die Möglichkeit,

- die Sterbehilfeorganisation im Psychiatriezentrum zuzulassen;

- die Patientin an einen geeigneten Ort (z. B. nach Hause) zu entlassen, wohin dann die Sterbehilfeorganisation kommen könnte.

\section{Ethische Überlegungen}

Dem Prinzip der Autonomie der Patientin wäre bei dieser Lösung optimal entsprochen, vor allem auch im Hinblick darauf, dass allgemein dieser Wunsch als «Therapiefortschritt» in dem Sinne 
angesehen wird, dass die Patientin Autonomie in dieser Frage beansprucht. Auch dem Prinzip der Gerechtigkeit wäre Rechnung getragen, indem psychisch Kranken die gleichen Möglichkeiten wie nicht psychisch Erkrankten dann eingeräumt werden, wenn der Grund für den Suizidwunsch nicht in Zusammenhang mit der psychischen Störung steht. Der Patientin würde in der Tat mit einiger Wahrscheinlichkeit eine recht quälende Krankheitsperiode erspart bleiben. Aus Sicht des Kollektivinteresses (andere psychisch Kranke, Psychiatriezentrum, allgemein psychiatrischer Kontext) ergibt sich aber eine differenziertere Problematik: mit einer Häufung von Suizidversuchen und Suiziden wäre gerade auch deshalb zu rechnen, da die Patientin im Psychiatriezentrum bekannt ist. Zumindest die Ärzteschaft des Psychiatriezentrums stände in Fachkreisen isoliert da, hätte mit massivsten Anfeindungen zu rechnen, was die Möglichkeit, weiterhin als glaubwürdige und qualifizierte Behandlungsstätte zu gelten, gefährden würde. Längerfristig könnte auch so mit einer Entwicklung gerechnet werden, in der (psychisch) Kranke auch vermehrt dann unter Druck gesetzt werden, diesen Schritt zu tun, wenn sie zu einer Belastung z.B. für Angehörige oder im schlimmsten Fall - leider aber nicht ganz abwegig (siehe Tötungen in Pflegeheimen) - auch für das Personal werden sollten. Das Psychiatriezentrum wäre in Gefahr, sich einen fatalen Ruf als Klinik, in der Sterbehilfe möglich ist, zu erwerben.

2. Das Psychiatriezentrum untersagt die Durchführung des Anliegens in jedem Fall mit dem obengenannten Argument; allenfalls stellt sie sich auch auf den - in der Psychiatrie nach wie vor weitverbreiteten - radikalen Ansatz, dass jeglicher Suizid wohl als Ausdruck von seelischer Not und Krankheit anzusehen ist, und ein Arzt verpflichtet, den Patienten daran zu hindern. Gerade bei einer abhängigen pflegebedürftigen Frau wäre so etwas auch prinzipiell durchführbar; an der Betreuung würde sich nichts ändern, da die Patientin sowieso in ihrer Bewegungsfreiheit eingeschränkt ist und in einer psychiatrischen Klinik auf einer Pflegeabteilung optimal betreut werden kann. Gleichzeitig müsste mit optimal palliativen Massnahmen gewährleistet sein, dass die Patientin möglichst wenig leidet. Auch das Beziehungsangebot durch den Therapeuten könnte so formuliert werden, dass er bereit ist, sie mit seinem ganzen Einsatz zum Tode zu begleiten, nicht aber mittels eines assistierten Suizids, der allenfalls sogar in Widerspruch mit diesem Angebot steht. Auch gibt es klare Ansätze, die dem Arzt gebieten, gerade für das Leben und nicht für den Tod eines Patienten einzutreten.

\section{Ethische Überlegungen}

Nach dem Prinzip der Autonomie wäre die Patientin massiv eingeschränkt, man würde ihre abhängige Situation auch benützen, um sie an der Durchführung ihres Anliegens zu hindern. Die Glaubwürdigkeit des Therapeuten wäre allenfalls bei diesem Ansatz in Gefahr, vor allem würde das ganze gegen das Prinzip der Gerechtigkeit verstossen, indem ja anderen, nicht psychisch kranken und abhängigen Menschen dieses Anliegen ermöglicht wird. Man könnte sie ja sogar allenfalls daran hindern, die Klinik trotz ihrer Urteilsfähigkeit in bezug auf die Frage des assistierten Suizides zu verlassen. Andererseits könnte man auf diese Art mittels eines Austrittsgesuches der Patientin die ganze Frage dem Gericht zur Beurteilung übergeben (Überprüfung der Hospitalisierungsbedürftigkeit gegen den Willen bei einer psychisch Kranken). Die Gefahr von Nachfolgesuiziden wäre gemindert, so dass gerade das Kollektivinteresse besser gewahrt werden könnte. Dem Personal würden in dieser Frage klare Leitlinien im Umgang mit allfällig weiteren Anliegen dieser Art gegeben werden. Für Chefarzt wie Regierungsrat wäre dies auch eine optimale politische Lösung, beide wären ihrer Verantwortung enthoben.

3. Zwischen diesen beiden Polen gäbe es noch ein diversifiziertes Vorgehen als Lösungsmöglichkeit: zunächst einen (allenfalls definierten) Zeitraum abwarten im Sinne eines Moratoriums zur Prüfung der Konsistenz dieses Wunsches; gleichzeitig optimale Palliativbehandlung, Beziehungsangebote, optimale Betreuung, alle Möglichkeiten nutzen, um der Patientin die Lebensqualität zu gewährleisten. Der Patientin gegenüber könnte auch vertreten werden, dass bei Persistenz ihres Suizidwunsches über den definierten Zeitraum hinaus die Verantwortlichen zumindest alles daransetzen würden, ihr dies wie auch immer zu ermöglichen oder ihr zumindest in der Durchführung nicht im Wege zu stehen; so wäre die Glaubwürdigkeit des Therapeuten gesichert, er hat «das getan, was er konnte».

\section{Ethische Überlegungen}

Dem Prinzip der Autonomie wäre nicht unmittelbar entsprochen, jedoch könnte als durchaus angemessen angesehen werden, wenn ein Moratorium einschaltet wird, verbunden mit den entsprechenden Begleitmassnahmen zur Steigerung der Lebensqualität. So wäre das Individualinteresse im Sinne der Autonomie zwar eingeschränkt, jedoch in vertretbarem Ausmass. Dem Kollektivinteresse wäre zumindest vorübergehend Beachtung geschenkt, das Vorgehen könnte als 
klare Leitlinie bei weiteren Fällen dienen und entspräche dem allgemeinen Vorgehen bei Suizidalen, indem auf die Moratoriumspflicht (selbst gegen Willen und Widerstand des Patienten) immer wieder auch verwiesen wird bei prinzipieller Anerkennung der Legitimität eines konsistenten und nicht krankheitsbedingten Suizidwunsches.

\section{Unter veränderten Voraussetzungen ...}

\section{Annahme}

Die Patientin wäre nicht psychisch krank und befinde sich deshalb nicht in einer psychiatrischen Institution, sondern in einem Pflegeheim. Unter diesen Voraussetzungen würde die Patientin die Bedingungen, wie sie vom Zürcher Stadtrat gestellt wurden, vollumfänglich erfüllen, ausser der Tatsache, dass sie noch eine eigene Wohnung hat, in die sie auch zur Durchführung des assistierten Suizides verlegt werden könnte. Die psychiatriespezifischen Dilemmata könnten so umgangen werden.

\section{Annahme}

Die Patientin wäre zwar psychisch krank, befinde sich aber zu Hause mit spitalexterner Pflege. Unter diesen Voraussetzungen wäre es ihr freigestellt, eine Sterbehilfeorganisation kommen zu lassen; niemand könnte ihr das verbieten. Die Sterbehilfeorganisation wäre für die Abklärung, ob Urteilsfähigkeit besteht, verantwortlich. Die Gefahr von Nachfolgesuiziden wäre zwar prinzipiell gegeben, aber nicht vermeidbar, sicherlich aber vermindert im Gegensatz zu einem stationären Aufenthalt.

\section{Entscheid des Chefarztes}

Der Chefarzt entschied, den Wunsch der Patientin aus den genannten Gründen aufzunehmen. Die Beteiligten wurden aber angewiesen, die Patientin lediglich in ihrer Entscheidungsfindung zu unterstützen, keinesfalls in irgendeiner Richtung Empfehlungen vorzunehmen. Ein Moratorium zur Prüfung der Konsistenz des Wunsches wurde vereinbart; die intensive Betreuung sollte weitergeführt werden, explizit auch im Hinblick auf eine optimale Unterstützung der Lebensqualität. Die ärztliche Behandlung nach palliativen Zielen sollte mit Zuhilfenahme von kompetenten Schmerzspezialisten erfolgen.

Die Situation entwickelte sich so, dass sich die Patientin mit diesem Ansatz offensichtlich ernst- genommen fühlte, die psychologische Betreuung wurde weiterhin intensiv und erfolgreich gestaltet, ebenso die Pflege. Mit Hilfe palliativer Massnahmen konnte die Patientin so behandelt werden, dass sie zwar immer wieder auch sehr stark unter Schmerzen litt, teilweise auch unter depressiven Zuständen, der Suizidwunsch wurde jedoch nicht wiederholt, nach einigen Wochen formulierte sie sogar explizit den Wunsch, nun den natürlichen Ausgang abzuwarten bzw... zu warten, bis «der Herrgott sie ruft».

Die Patientin verstarb in Anwesenheit einer der Bezugspersonen.

\section{Offene Fragestellungen}

Sie können meinen Ausführungen entnehmen, dass - unabhängig von welcher Seite her man diesen Fall betrachtet - ein gewisses Unbehagen bleibt: wie weit können, dürfen, sollen wir uns auch im therapeutischen Gespräch auf die Wünsche nach Sterbehilfe vorwagen, sie auf- und ernstnehmen, wo ist die Grenze hin zur Unterstützung $\mathrm{zu}$ einem solchen Schritt? Wo die Grenze hin zu nicht mehr gerechtfertigter Autonomieeinschränkung? Wo werden Entscheide aus blossem Opportunismus, um die Verantwortung zu delegieren, getroffen oder auch nicht getroffen? Stellt das hier vorgeschlagene Moratorium nicht auch einen Versuch dar, unangenehme Entscheide zu vertagen, bis sie sich «selbst gelöst haben», auf die Gefahr hin, dass die Patientin die Botschaft versteht, ihr Anliegen nicht mehr formuliert und andere Wege sucht? Gehen wir davon aus, dass Patientinnen und Patienten mit einer infausten somatischen Diagnose diesen Schritt unternehmen dürfen, ist bei psychisch Kranken dies keinesfalls möglich aufgrund der beschriebenen Problematik? Sind klare, eindeutige Positionen (z.B. kategorische Ablehnung der Durchführung, Anerkennung, Unterstützung eines Sterbewunsches) nicht einfacher zu handhaben, wo ist die Grenze, die die Gesellschaft noch tolerieren kann und sollte?

Mir ist es ein Anliegen, zu betonen, dass die verschiedenen Argumente nicht in jedem Fall die Meinung des Autors wiedergeben, es sind Versuche, sich im Rahmen eines ethischen Diskurses der Materie anzunähern. Auch bin ich nicht der Meinung, dass der dann beschrittene Weg der einzig richtige, mögliche, der «weise Weg» ist, und ich bin mir auch darin bewusst, dass gerade die Psychiatrie von ihrer Geschichte nicht losgelöst betrachtet werden kann, ebenfalls, dass gesellschaftliche Entwicklungen vermeintliche «gültige Aussagen» rasch einmal auch verändern können. 


\section{Der Tod als Freund}

\author{
Suizidwunsch bei einer psychiatrisch erkrankten Patientin
}

\section{R. Baumann-Hölzle}

Die folgende Fallbesprechung für Frau M. basiert auf dem System der 7 Schritte ethischer Urteilsbildung von Dialog Ethik. Sie strukturieren den Entscheidungsfindungssprozess, ohne den Entscheid selbst vorwegzunehmen. Ausgegangen wird dabei vom Nichtwissen aller Beteiligten bezüglich der endgültigen Entscheidung am Anfang des Entscheidungsfindungsprozesses. Ziel dieses Weges ist ein Entscheid, der sowohl in individualethischer als auch sozialethischer Hinsicht verantwortet werden kann.

\section{Schritt: Ethische Kernfragen}

Die beschriebene Situation von Frau M. wirft individual-, personal- und sozialethische Fragen auf: Darf Frau M. mit einer schlechten Langzeitperspektive und hohem Leidensdruck die Suizidbeihilfe im Rahmen einer psychiatrischen Institution verweigert werden, weil diese entweder von Beteiligten persönlich abgelehnt oder der Wunsch nach einem Suizid als krank eingestuft wird (individual- und personalethische Fragestellung) oder weil befürchtet wird, dass daraus Zwänge für andere Patientinnen und Patienten in vergleichbaren Abhängigkeitssituationen (sozialethische Fragestellung) resultieren können? Bei der Frage nach der Legitimität der Suizidbeihilfe für Frau M. stehen die Reichweite des individuellen Autonomieanspruchs und die soziale Verantwortung aller Beteiligten gleichzeitig zur Debatte.

\section{Schritt: Kontextanalyse}

Bei der Kontextanalyse wird ein möglichst umfassendes Bild von der zu beurteilenden Situation erhoben und nach noch ausstehenden Informationen gefragt.

Es ist anzunehmen, dass der Suizidwunsch von Frau M. eine Reaktion auf die infauste Prognose ist, welche ihre Ohnmachtsgefühle gegenüber ihrer Situation, in der die Abhängigkeit dominiert, massiv verstärkt. Mit dem Suizidwunsch will sie Macht über ihre eigene Lebenssituation gewinnen. Wichtig wäre zu wissen, was «infauste Prognose» bei diesem Karzinom konkret bedeutet: Handelt es sich dabei über einen kurzen oder langen Zeitraum, in dem die Frau noch leben kann oder nicht, und was hat sie dabei zu erwarten? Die Fallbeschreibung gibt explizit keine Auskunft darüber, ob Frau M. den Suizidwunsch über einen längeren Zeitraum mehrmals und ob sie ihn ausschliesslich gegenüber dem Psychologen oder auch anderen Personen gegenüber in gleicher Art und Weise geäussert hat. Die Beziehung der Patientin zum Psychologen scheint sehr eng zu sein. Sie wäre genauer zu betrachten. Ebenfalls wissen wir nicht, ob die Patientin in der Vergangenheit ebenfalls Suizidwünsche formuliert hat und - falls dem so ist - wie diese jeweils verlaufen sind. Zudem ist zu fragen, inwieweit die Klinik Erfahrungen mit palliativer Begleitung und Betreuung hat: Ist konkret Fachwissen in Palliative Care vorhanden? Wir wissen zu wenig über weitere Bezugspersonen ausser dem Ehemann: Hat Frau M. Kinder? Gibt es noch weitere Menschen, die ihr nahestehen? Wer hat sie bisher ausserhalb der Klinik durch Lebenskrisen begleitet? Diese Informationen würden nun bei einer regulären Fallbesprechung in den Entscheidungsfindungsprozess einfliessen. In dieser Situation müssen wir uns mit Mutmassungen zufriedengeben.

\section{Schritt: Ethische Dilemmata}

Ein ethisches Dilemma liegt dann vor, wenn man bei einem Entscheid nicht umhinkommt, eine ethisches Prinzip zu verletzen. In der Bioethik werden folgende vier Prinzipien gegeneinander abgewogen: das Autonomieprinzip, das Prinzip, nicht zu schaden, das Prinzip, Gutes zu tun, und das Gerechtigkeitsprinzip. Angesichts der schlechten Langzeitperspektive und des hohen Leidensdruckes kann der Tod bei Frau M. nicht mehr nur als Schaden qualifiziert werden, den es zu verhindern gilt. Individualethisch stehen sich bei Frau M. das Autonomieprinzip (Anspruch auf Suizidbeihilfe) und das Prinzip, Gutes zu tun, (die Betreuenden lehnen die Suizidbeihilfe aus Gewissensgründen ab und/oder qualifizieren im Gegensatz zu Frau M. andere Massnahmen als «gut») gegenüber.

Sozialethisch steht das Gerechtigkeitsprinzip in zweifacher Hinsicht gegenüber dem Autonomieprinzip auf dem Spiel. Erstens, darf man der Frau M. unter den gegebenen Lebensumständen verweigern, was ihr ausserhalb der Klinik möglich wäre, und zweitens, darf man Frau M. etwas zugestehen, was unter Umständen Autonomieverletzungen von andern Menschen zur Folge hätte, wenn Suizidbeihilfe zum sozialen Zwang wird? 


\section{Verhaltensmöglichkeiten}

Nach den ersten drei Analyseschritten gilt es, immer mehr als zwei Verhaltensoptionen tabulos im Brainstorming zu formulieren:

1. dem Wunsch von Frau M. als Wille zu entsprechen;

2. Frau M. den Wunsch zu verweigern;

3. Beizug von externen, unabhängigen Gesprächspartnern;

4. dem Wunsch von Frau M. erst nach einem Moratorium Folge zu leisten;

5. alle leidenslindernden Massnahmen auszuschöpfen und dann dem Wunsch von Frau M. zu entsprechen;

6. Verlegung von Frau M. nach Hause;

7. Verlegung von Frau M. in eine andere Klinik.

\section{Schritt: Gesetzeslage, Richtlinien und Ethikentwürfe}

Vom Gesetz her sind in der Schweiz alle Handlungsvorschläge möglich. Die Suizidbeihilfe ist in der Schweiz eine Freiheit und kein Recht, d. h. niemand kann Suizidbeihilfe als Recht einfordern. Die Suizidbeihilfe ist straffrei, wenn sie aus Mitleid und ohne eigennützige Zwecke geschieht. Diese Straffreiheit soll demjenigen, der aus einer inneren Not und Mitleid einem ihm nahestehenden Menschen gegenüber im Namen der Gewissensfreiheit Beihilfe leistet, gewährt werden.

Soeben sind die «Medizinisch-ethischen Richtlinien und Empfehlungen zur Behandlung und Betreuung von älteren pflegebedürftigen Menschen» der Schweizerischen Akademie der Medizinischen Wissenschaften in die allgemeine Vernehmlassung gegeben worden. Dort werden klare Aufgaben und Bedingungen an die Institutionen gestellt, wenn ein Mensch einen Suizidwunsch äussert. So wird unter anderem verlangt, dass die Betreuungssituation des betroffenen Menschen genau angesehen und auch von einem aussenstehenden, unabhängigen Arzt überprüft wird. Es stellt sich die Frage, ob diese Richtlinien nicht auch für psychiatrische Institutionen Geltung haben können.

Was die Ethikentwürfe anbelangt, so ist es interessant zu bemerken, dass der Philosoph I. Kant, der als erster den Begriff der «Autonomie» verwendet hat, den Suizid explizit als Selbstinstrumentalisierung ablehnt. Hingegen wird von utilitaristischen Ethikentwürfen, bei denen Kosten-Nutzen-Überlegungen im Zentrum stehen, die Freiheit zum Suizid und zur Suizidbeihilfe unterstrichen.

\section{Schritt: Grundsatzüberlegungen}

Bevor die einzelnen Handlungsmöglichkeiten gegeneinander abgewogen werden und eine Entscheidung gefällt wird, gilt es, die Frage der Suizidbeihilfe in dieser Institution, in denen sich Menschen in besonderen Abhängigkeitssituationen befinden, grundsätzlich und unabhängig von Frau M. zu bedenken: Wie möchte man sich im Psychiatriezentrum Schaffhausen allgemein gegenüber dem Wunsch nach Suizidbeihilfe bei Patientinnen und Patienten verhalten? Dabei gilt es, die Verantwortlichkeiten gegenüber Frau M., die Verantwortlichkeiten von Frau M., die Verantwortlichkeiten gegenüber der Institution und die gesamtgesellschaftliche Verantwortung zu bedenken, wie dies Herr Ebner in seiner Fallbesprechung sehr sorgfältig tut.

\section{Individualethische Überlegungen}

In der medizinethischen Urteilsbildung hat ein grundsätzlicher Paradigmenwechsel in der Entscheidungsfindung weg von der Orientierung ausschliesslich an der Lebenserhaltung der Patientin hin zur Patientenautonomie stattgefunden. Dies, weil einerseits die medizinischen Möglichkeiten zur Lebenserhaltung selbst Leiden verursachen können, und andererseits in einer pluralistischen Gesellschaft Menschen nicht mehr den gleichen Sinnhorizont haben. In diesem Kontext gilt jede medizinische Handlung als Körperverletzung und bedarf der informierten Zustimmung («informed consent») durch die Patientin. Autonomie in diesem Sinne ist ein Abwehrrecht, welches die Menschen vor Übergriffen und Instrumentalisierung durch andere Menschen schützen soll. Der normative Autonomieanspruch besteht sogar dann, wenn ein Mensch selber nicht mehr urteilsfähig ist: In dieser Situation ist gemäss dem mutmasslichen Willen des Urteilsunfähigen zu handeln. Dieser immer interdisziplinär unter Einbezug der nächsten Bezugs- und Beziehungspersonen zu erheben. Hierfür sind die notwendigen Rahmenbedingungen von Raum und Zeit zur Verfügung zu stellen.

Dieser normative Autonomieanspruch ist von den tatsächlichen Autonomiefähigkeiten eines Menschen $\mathrm{zu}$ unterscheiden: Eingeschränkte Autonomiefähigkeiten legitimieren keine Übergriffe, sondern der normative Autonomieanspruch verpflichtet die Beteiligten, einem Menschen trotz seiner Einschränkungen informierte Entscheide zu ermöglichen.

Was aber bedeutet «informed consent» im Kontext von einseitigen Abhängigkeitsbeziehungen? Die Autonomiefähigkeiten eines Menschen 
hängen stark von den jeweiligen Lebensumständen ab. Ein informierter Entscheid kann deshalb nur nach Kenntnisnahme von alternativen Lebensmöglichkeiten getroffen werden. Autonomie und Urteilsfähigkeit sind deshalb nicht ein punktuelles Ereignis, sondern ein Prozess, der Zeit braucht: Dabei werden die verschiedenen Handlungsmöglichkeiten gegeneinander auf ihre Angemessenheit hin abgewogen. In diesem Prozess erweist sich ein Mensch als verantwortliches Subjekt. Autonomie und Verantwortung gehören untrennbar zusammen. Wunsch und Wille eines Menschen sind deshalb auseinanderzuhalten: Was ursprünglich als Wunsch in Erscheinung tritt, kann sich nach reiflicher Überlegung als irrelevant zeigen oder gar dem Willen widersprechen. Bei alltäglichen Entscheidungen stehen solche Willensbildungsprozesse im freien Belieben eines Menschen.

Demgegenüber wird bei Patienten, die nicht irreversibel in absehbarer Zeit sterben werden, die Lebenserhaltung vor allem im Notfall höher gewichtet als ihr Todeswunsch. Deshalb werden in der Regel Menschen nach einem Suizidversuch am Leben erhalten und werden Menschen daran gehindert, sich bewusst Selbstverletzungen zuzufügen. Die Legitimität des fürsorgerlichen Freiheitsentzugs in der Psychiatrie basiert auf dieser Wertentscheidung. Ihr Todeswunsch wird als nicht ihrem Willen entsprechend eingestuft. In dieser Situation wird der Tod als Schaden qualifiziert. Das Prinzip der Schadensvermeidung legitimiert dabei die Verletzung des Autonomieprinzips.

Leidet ein Mensch an einer Krankheit, die trotz medizinischer Interventionen irreversibel in absehbarer Zeit den Tod bringen wird, wird der Suizid nicht mehr verhindert und die Suizidbeihilfe auf individualethischer Ebene zunehmend auch innerhalb von Institutionen toleriert. Damit zeichnet sich ein weiterer Paradigmenwechsel ab: Der Mensch will völlig frei über seine eigene Leiblichkeit verfügen. Damit entfällt jegliche Selbstverantwortung gegenüber der eigenen Leiblichkeit zu deren Erhaltung und Pflege. Mit dieser Instrumentalisierung des Leibes durch den Willen kann gegen Selbstschädigung und Selbstzerstörung nicht mehr eingegriffen werden. Zwangspsychiatrische Interventionen sind dann nur noch im Falle der Fremdgefährdung möglich.

\section{Sozialethische Überlegungen}

Zurzeit findet ein Gesinnungswechsel statt: Die Freiheit zum Suizid und zur Suizidbeihilfe wird zunehmend zum moralischen Anspruch und zum einforderbaren Recht gegenüber anderen Menschen. Die Option der Tötung gelangt so in den sozialen Kontext. Damit verliert die Beihilfe zur Selbsttötung ihren privaten Charakter. Sie muss gesamtgesellschaftlich verantwortet werden.

Untersuchungen in den Niederlanden zeigen: Der Wunsch nach Tötung erwächst meist nicht aus unheilbarer Krankheit oder starken Schmerzen, sondern aus dem Wunsch, Abhängigkeitssituationen entfliehen zu können. Je mehr Unterstützung kranke Menschen für eine selbständige Lebensführung erhalten, umso weniger taucht der Todeswunsch auf. Ob diese Unterstützung Menschen in ausgeprägten Abhängigkeitssituationen auch in Zukunft gewährt werden wird, bleibt dahingestellt. Bedenkt man die Finanzierungsprobleme im Pflegebereich einerseits und die demographische Entwicklung andererseits, so stellt sich die Frage, ob nicht eines Tages die Suizidbeihilfe gesamtgesellschaftlich als einfache und kostengünstige Problemlösung für schwierige Pflegesituationen angesehen wird.

Wegen der Schutzverpflichtung schwachen Menschen gegenüber bedarf es deshalb verbindlicher gesetzlicher Regelungen. Es kann nicht dem Belieben der einzelnen Institutionen überlassen bleiben. Die Richtlinien der SAMW setzen hierfür einen verbindlichen Standard. Ob mit solchen Regelungen der soziale Druck auf chronischkranke Menschen, der Gesellschaft nicht zur Last zu fallen, aufgefangen werden kann, wird sich weisen. Wie andere Klimaveränderungen vollziehen sich auch gesellschaftliche Klimaveränderungen langsam und schleichend. Oft werden sie erst bemerkt, wenn der Schaden schon da ist.

Der Suizid und die Suizidbeihilfe werden im Namen der Autonomie im öffentlichen Raum eingefordert. Damit trägt auch der Suizidwillige eine soziale Verantwortung dafür, dass durch seine Handlung andere Menschen nicht unter Druck geraten, es ihm gleichtun zu müssen. Diese soziale Verantwortung ist umso grösser, je öffentlicher der Raum ist, in dem er sich befindet. Der Rückzug in private Räume, falls diese vorhanden sind, für die Durchführung eines Suizids ist ihm deshalb genauso zuzumuten wie eine Wartezeit, während der er alle anderen Handlungsmöglichkeiten prüfen und ausschöpfen kann. Gleichzeitig besteht auf seiten der Institution die Verpflichtung, die Lebensumstände eines Menschen optimal zu gestalten. 


\section{Schritt: Evaluation des getroffenen Entscheides}

Kehren wir zu Frau M. zurück. Für eine abschliessende Evaluation des in Schaffhausen sehr sorgfältig und bewusst getroffenen Entscheides fehlen verschiedene Informationen (s. Kontextanalyse). Vor allem die Information darüber, wie sich Frau M. in der Vergangenheit in bezug auf Suizdwünsche verhalten hat, wäre wichtig.

Tatsache ist, dass bei Frau M. die Änderung ihrer Lebensumstände zu einer Haltungsänderung bezüglich ihres Todeswunsches geführt hat. Offenbar waren zum Zeitpunkt des Suizidwunsches die Möglichkeiten zur Verbesserung ihrer Lebensqualität nicht ausgeschöpft gewesen.

Die soziale Verantwortung von Frau M. legitimiert meines Erachtens das Zeitmoratorium innerhalb der Klinik. Falls sie damit nicht einverstanden gewesen wäre, hätte sie die Möglichkeit gehabt, die Klinik zu verlassen. Das Moratorium verpflichtet aber die Behandelnden und Betreuenden dazu, die Verbesserungsmöglichkeiten der Lebensumstände von Frau M. auszuschöpfen. Dies ist auch geschehen.

Frau M. ist von ihrem Umfeld respektiert und ernst genommen worden und damit hat sich ihr Suizidwunsch verflüchtigt. Dieser Grundrespekt ist Voraussetzung dafür, dass Menschen auch in ausgeprägten Abhängigkeitssituationen die Behandlung und Betreuung bekommen, die ihnen zustehen. Für einen sogenannt «freien Entscheid» gegen das eigene Leben ist das Ausschöpfen guter Lebensumstände Bedingung. Sicher ist, dass sich unter solchen Umständen nur sehr wenig Menschen vorzeitig aus ihrem Leben verabschieden wollen.

\section{Schlussgedanken}

Lässt sich der Tod mit lebenserhaltenden Massnahmen irreversibel nicht mehr vermeiden, wird er vom Feind zum Freund. Der Freund kommt, wann er will. Er lässt sich nicht herbeizwingen. Zwang ist einer Freundschaft nicht würdig, sie lebt von der Freiheit. Im gemeinsamen geduldigen Warten auf den Tod wird das gelebte Leben plastisch im Hinblick auf das, was zählt. Die Wartenden werden zur solidarischen Gemeinschaft, die über den Tod hinaus Bestand hat. Für diese Lebensgemeinschaft übernimmt der Sterbende mit seinem Warten zum letzten Mal Verantwortung und erweist sich gerade in diesem Dienst an der Gemeinschaft als autonomes Subjekt. 


\title{
Schutz der Institution vorrangig
}

\author{
G. Bosshard
}

1 Birnbacher D. Selbstmord und Selbstmordverhütung aus ethischer Sicht. In: Leist A (Hrsg.). Um Leben und Tod. Moralische Probleme bei Abtreibung, künstlicher Befruchtung, Euthanasie und Selbstmord. Frankfurt a. M: Suhrkamp; 1990. S. 395-422.

2 Emanuel EJ, Fairclough DL, Emanuel LL. Attitudes and desires related to euthanasia and physician-assisted suicide among terminally ill patients and their caregivers. JAMA 2000;284:2460-8.

3 Back AL, Starks H, Hsu C, Gordon JR, Bharucha A, Pearlman RA. Clinician-patient interactions about requests for physicianassisted suicide. A patient and family view. Arch Int Med 2002; 162:1257-65.

Korrespondenz:

Dr. med. Georg Bosshard, MAE

Institut für Rechtsmedizin

Universität Zürich-Irchel

Winterthurerstrasse 190/ Bau 52

CH-8057 Zürich

E-Mail: bosh@irm.unizh.ch
Kann es rationale Motive geben, um sich selber das Leben zu nehmen? Diese Frage ist, gerade in der Psychiatrie, aufs heftigste umstritten. Der Fallbericht von Gerhard Ebner trifft mit seiner eindrücklichen, lebensnahen, Einseitigkeiten vermeidenden Schilderung unmittelbar den Kern dieser Debatte. Und auch der mit dem Alltag einer psychiatrischen Klinik wenig vertraute Leser mag nachvollziehen, was für ein enormes Spannungsfeld sich eröffnet, wenn im psychiatrischen Umfeld Tätige sich nicht, wie sonst üblich, einem Ersuchen um Hilfe beim Suizid kategorisch entgegenstellen.

Dabei handelt es sich ja um einen für eine psychiatrische Klinik durchaus untypischen Fall: Eine wegen eines Karzinoms zunehmend pflegebedürftige Frau wird auf einer psychiatrischen Pflegeabteilung stationär behandelt. Die Hospitalisation in dieser Institution erfolgt offenbar weniger deshalb, weil akute psychiatrische Symptome vorhanden gewesen wären, als vielmehr, weil die Patientin im Rahmen ihrer langjährigen depressiven Erkrankung das Psychiatriezentrum als Zufluchtsort schätzengelernt hat. Plausibel wird geschildert, wie der Sterbewunsch der Patientin diesmal deutlich anders, für den Beobachter nachvollziehbarer, motiviert ist, als es früher im Rahmen depressiver Krankheitsphasen der Fall war.

Der deutsche Philosoph Dieter Birnbacher hat vorgeschlagen, sich in Situationen wie der vorliegenden vom Gedanken leiten zu lassen, «dass jemand, der einen Selbstmord begehen will, immer dann mit allen Mitteln, notfalls auch mit Gewalt, von seinem Vorhaben abzuhalten ist [...], wenn zu erwarten ist, dass der Betroffene die Vereitelung seiner Tat später billigen wird» [1]. An einer anderen Stelle spricht er von einer «wohlerwogenen Entscheidung zum Suizid» als einer der Bedingungen für die moralische Zulässigkeit von Suizidbeihilfe. Wichtig ist in diesem Zusammenhang, dass auch bei terminal kranken Menschen Sterbewünsche bzw. das Verlangen nach Sterbehilfe oftmals instabil sind. Das gilt insbesondere dann, wenn ein Patient zusätzlich depressive Symptome aufweist [2].

Ein Moratorium, also eine Wartefrist unter Beizug palliativ-medizinischer Experten zur Bekämpfung der ausgeprägten Schmerzen derart, wie es im Fallbeispiel gewählt wurde, scheint mir persönlich somit ein vernünftiges Vorgehen. Der Sterbewunsch wird ernstgenommen und quasi «verhandelt», aber nicht von vorneherein kategorisch abgelehnt. Kürzlich wurde in einer Studie, welche die Sicht von sterbewilligen Patienten und deren Angehörigen untersucht hat, auf eindrückliche Weise gezeigt, wie eine kategorische Ablehnung solcher Wünsche durch den Arzt oftmals das Vertrauensverhältnis zum Patienten gefährdet, während umgekehrt eine nicht vorschnell wertende Haltung dasselbe festigen kann [3]. Dass dabei die jeweilige Reaktion des Arztes auch ein Spiegelbild seiner eigenen Einstellung zum Tod, seiner diesbezüglichen Zweifel und Ängste darstellt, ist offensichtlich.

Ob sich nun allerdings diese Überlegungen zur Sterbehilfe bei körperlich unheilbar Schwerstkranken oder Sterbenden auch auf Personen übertragen lassen, deren Sterbewunsch durch ein psychisches Leiden bedingt ist, erscheint zweifelhaft. Zum einen lassen die grundsätzlichen Unsicherheiten in der längerfristigen Prognose psychiatrischer Leiden einen Sterbewunsch oftmals auch dann nicht als wohlerwogen erscheinen, wenn juristisch die Urteilsfähigkeit des Sterbewilligen gegeben sein sollte. Zum anderen - und dieser Einwand ist vielleicht der gewichtigere von beiden - kann doch wohl kaum im Ernst erwartet werden, dass in psychiatrischen Kliniken Tätige, welche über Jahre und vielleicht Jahrzehnte gegen die wiederkehrende Suizidalität eines psychisch kranken Menschen angekämpft haben, zu einem gewissen Zeitpunkt plötzlich ihre Bemühungen einstellen und das Gegenteil tun sollen, nämlich ihrem Patienten beim Suizid helfen. Vor solchen Forderungen müssen deshalb psychiatrische Institutionen geschützt werden. Andernfalls würden deren grosse Bemühungen zur Verhinderung von Suiziden psychisch kranker Menschen ernstlich in Frage gestellt.

Dies ist nun nicht primär ein Argument gegen Sterbehilfe bei psychisch Kranken als vielmehr eines gegen Suizidbeihilfe in psychiatrischen Institutionen. Es gilt auch im beschriebenen Fall. Das bedeutet, dass selbst dann, wenn die Patientin nach Ablauf der Wartefrist bei ihrem Sterbewunsch geblieben wäre, eine Beihilfe zum Suizid keine ernsthafte Option für die in der Klinik Tätigen hätte sein können. Es wäre dann wohl eher darum gegangen, sich den Bemühungen der Patientin, den Freitod mit Hilfe einer Sterbehilfeorganisation bei sich zu Hause zu organisieren, nicht entgegenzustellen. Auch das Rezept für die tödlich wirksame Substanz sollte in diesem Fall von einem externen Arzt ausgestellt werden.

Beihilfe zum Suizid durch eine Institution, zu deren Hauptaufgaben das Verhindern von Suiziden gehört, scheint mir persönlich in jedem Fall ein Unding. 


\title{
Stellungnahme zum Fall einer psychisch Kranken mit einer unheilbar körperlichen Krankheit mit Sterbewunsch
}

\author{
B. Küchenhoff, D. Hell
}

Korrespondenz:

Dr. med. B. Küchenhoff

Psychiatrische Universitätsklinik

Lenggstrasse 31

CH-8008 Zürich
Die vorliegende Falldarstellung behandelt eine wichtige Fragestellung in offener und klarer Weise. Dabei werden auch ethische Überlegungen im Hinblick auf den Sterbewunsch einer körperlich schwer kranken Frau, die an Depressionen leidet, gemacht.

In unserer Stellungnahme gehen wir davon aus, dass es vorrangige Aufgabe des Arztes ist und bleibt, Krankheiten $\mathrm{zu}$ diagnostizieren und $\mathrm{zu}$ behandeln sowie Leiden zu lindern. Dazu gehört, wie im konkreten Beispiel, auch eine bestmögliche Sterbebegleitung. Wir sind allerdings der Auffassung, dass die Unterstützung eines Sterbewunsches keine ärztliche Aufgabe ist. Gerade wenn man die Autonomie eines Patienten hoch einschätzt, ist ein Sterbewunsch ernst zu nehmen, ohne ihn zu fördern. Im Fallbericht befürchtet die Patientin, qualvoll zu sterben und äussert den Wunsch, ihr Leben aktiv zu beenden. Die im Fallbericht erwähnte Reaktion der Therapeuten, mit der Patientin über ihr Leiden und über ihren Todeswunsch offen $\mathrm{zu}$ sprechen, ist sicher zu begrüssen. Unseres Erachtens darf aber nicht der Eindruck erweckt werden, dass der behandelnde Psychiater oder gar die psychiatrische Klinik als Institution einem solchen Wunsch entsprechen kann.

Die Patientin hat sich im vorliegenden Fall allerdings nicht an die Ärzte, sondern an ihren Psychologen gewandt. Dieser hatte einen sehr engen Kontakt zu der Patientin, den er auch während seiner Ferien aufrechterhielt. Die Patientin, die als zurechnungsfähig beurteilt wird, wusste sicher, dass der Psychologe nicht selbst über die entsprechenden Mittel zur Tötung verfügte. Deshalb wollte sie über ihn auch an eine Sterbehilfeorganisation gelangen. Sie wünschte also die Durchführung der Tötung durch ihr fremde Menschen und nicht etwa durch die ihr vertrauten Pflegenden und therapeutischen Personen.

Unerklärlich ist uns, dass der Psychologe gerade den Sterbewunsch als Ausdruck dafür wertet, «dass die Patientin sich von Abhängigkeitsbeziehungen lösen und ihr Leben bei einer existenziellen Frage aktiv in die Hand nehmen möchte». Er sah deswegen die Ablehnung dieses Wunsches als «Verrat» an. Warum sollte vor allem der Sterbewunsch als wesentlicher autonomer Akt der Patientin angesehen werden, den es zu unterstützen gilt? Hat die Patientin im Alltag nicht mit Entscheidungen $\mathrm{zu}$ ringen, die ihre Autonomie herausfordern?

In diesem Fall stellen sich auch institutionelle Fragen, die über die individuelle Problematik hinausgehen. $\mathrm{Zu}$ den im Fallbericht bereits angesprochenen Punkten möchten wir einen uns wichtig erscheinenden Aspekt hinzufügen:

Es ist schwierig, auf der gleichen Station einer psychiatrischen Klinik einerseits Patienten zu behandeln, die gegen ihren Willen wegen Suizidalität zurückgehalten werden und andererseits dem Sterbewunsch anderer Patienten nachzukommen. Auch unter Berücksichtigung der eugenisch besonders belasteten Geschichte der Psychiatrie sollten unseres Erachtens psychiatrische Kliniken alles daran setzen, zu verhindern, dass Patienten in der Klinik bzw. mit Hilfe der Klinik getötet werden. Im vorliegenden Fall stellt sich die Frage, ob für die Patientin, die weniger wegen ihrer Depression als ihres schmerzhaften Körperleidens in psychiatrischer Klinikbehandlung steht, nicht andere Lösungen zu prüfen sind, z. B. intensivere palliative Massnahmen.

Abschliessend möchten wir $\mathrm{zu}$ bedenken geben, dass bei jeder psychiatrisch-psychotherapeutischen Behandlung das Setting mit seinen Regeln einen wichtigen Bestandteil darstellt. Unseres Erachtens gehört zum Behandlungssetting einer psychiatrisch-psychotherapeutischen Einrichtung dazu, dass nicht aktiv Sterbehilfe geleistet wird. Wir halten diese Einstellung, die den Patienten auch transparent gemacht werden muss, gerade im Prozess der Sterbebegleitung für hilfreich und notwendig. Wenn Patienten andere Wege und eine andere Entscheidung für sich finden wollen, so hat niemand darüber zu richten.

Auch wenn wir auf den konkreten Ausgang dieses Falles nicht näher eingegangen sind, so scheint er doch unsere Haltung eher zu stützen. 